\title{
Philosophiques
}

\section{Théories et pratiques de la désaliénation}

\section{René Pellerin}

Volume 8, numéro 1, avril 1981

URI : https://id.erudit.org/iderudit/203149ar

DOI : https://doi.org/10.7202/203149ar

Aller au sommaire du numéro

Éditeur(s)

Société de philosophie du Québec

ISSN

0316-2923 (imprimé)

1492-1391 (numérique)

Découvrir la revue

Citer cet article

Pellerin, R. (1981). Théories et pratiques de la désaliénation. Philosophiques, 8(1), 49-74. https://doi.org/10.7202/203149ar d'utilisation que vous pouvez consulter en ligne.

https://apropos.erudit.org/fr/usagers/politique-dutilisation/ 


\title{
THÉORIES ET PRATIQUES DE LA DÉSALIÉNATION
}

\author{
par René Pellerin
}

\begin{abstract}
Mon projet ici en est un de désillusion. Il ne s'agit pas de paralyser les entreprises auxquelles donne lieu l'aliénation, mais de les situer dans leur contexte, de déterminer leurs limites et de préciser leur statut propre par rapport à leur objet. Diverses prétentions sont dissimulées mais très opérantes dans ces entreprises, qu'elles soient théoriques ou pratiques. Ces prétentions reposent sur des justifications ou des décisions dont les fondements nous sont apparus éminemment «flottants» et imprécis. Des ambitions factuellement constatées et des convictions sur lesquelles les initiateurs de théorisation ou de transformation sociopolitique n'ont pas de prise sont à l'oeuvre, mais sont presque systématiquement rejetées en dehors de la problématique de l'aliénation. Il s'agira ici de mettre au clair la position qui est implicitement privilégiée par les intervenants dans cette problématique.
\end{abstract}

Les entreprises de théorisation ou de thérapie de l'aliénation sont-elles incluses dans ce qu'elles prétendent atteindre comme de l'extérieur? L'aliénation est souvent l'occasion de réactions émotionnelles intempestives liées au scandale de la souffrance et de l'injustice, et ces réactions sont associées à un discours de justification qui fait appel à des "évidences» morales et à des impératifs catégoriques rien moins que dogmatiques. En critiquant ces évidences et ces appels au "sens moral», nous ne voulons pas réfuter la valeur des entreprises de désaliénation ou des entreprises de théorisation, mais plus modestement localiser les insuffisances de leur justification et les lacunes de leur argumentation. La valorisation qui est à l'oeuvre dans ces entreprises constitue davantage un fait de conscience qu'une justification qui serait finale. 
On peut en effet se demander, malgré la stupéfaction que cela peut provoquer, pourquoi faut-il ultimement faire ce qui nous apparaît de fait comme devant être fait ou valant la peine d'être fait, et quelle part ma liberté joue-t-elle dans la décision d'intervenir en faveur d'une valeur qui s'impose à moi malgré moi?

Je n'ai pas essayé de répondre ici à cette question, mais plutôt de montrer les conséquences en quelque sorte «humiliantes» de l'absence de réponse à ces questions lorsque des interventions théoriques ou pratiques ont tout de même lieu et lorsqu'elles sont présentées ou supposées justifiées "par elles-mêmes». Les théoriciens et les praticiens dans la «cause» de l'aliénation pourraient bien être en train de révéler leur propre aliénation lorsqu'ils interviennent plutôt que de la dépasser ou de la «régler».

Nous analyserons d'abord comment il y a visée de valeur dans la démarche théorique et dans la démarche pratique. Nous examinerons ensuite les difficultés et les impasses dans lesquelles se sont enlisés ceux qui ont essayé de fonder leurs interventions et ceux qui prétendent pouvoir "réussir» à désaliéner les autres sans s'être assurés de leur propre désaliénation. Nous proposerons enfin la question de la possibilité d'une intervention authentiquement désaliénante et nous suggérerons une voie de recherche qui nous mènerait à la découverte des conditions d'efficacité d'une telle intervention ${ }^{1}$.

\section{IL Y A UNE VISÉE THÉORIQUE}

L'examen, même superficiel, de la littérature scientifique, philosophique et politique concernant l'aliénation révèle deux directions qui sont la marque de la plupart des sciences humaines: la direction, ou mieux peut-être l'insistance, explicative ou théorique, et l'insistance performative ou pratique. Il ne s'agit pas d'une dichotomie où l'on pourrait séparer totalement l'une de ces insistances de l'autre, mais bien plutôt d'une ambivalence marquée par l'une plus que l'autre. Marx, Freud, Nietzsche et les sociologues de la connaissance depuis K. Mannheim ont bien montré l'impossibilité d'isoler, sauf abstraitement, la production intellectuelle de la situation concrète, sociale en particulier, des

1. Ce texte est une version remaniée et considérablement augmentée d'une communication présentée au congrès de l'Association canadienne de philosophie, tenu à l'UQAM en juin 1980. 
producteurs. Ceci cependant n'exclut pas une dualité; au contraire, celle-ci est reconnue dans le projet même d'une mise en relation d'interdépendance. Dans les pratiques théoriques et/ou pratiques s'exprimeront des «tendances» qui permettront de distinguer, pour la question de l'aliénation, une «science» de l'aliénation d'une «morale» de la désaliénation.

La distinction ne concerne pas ici le contenu des deux perspectives, mais plutôt leur orientation sur des valeurs différentes: la connaissance objective, d'une part, et la promotion de situations ou d'expériences existentiellement positives, d'autre part. Ces valeurs incluent à la fois la démarche et le produit qui «devrait» en résulter. Ce sont donc des valeurs qui sont différemment privilégiées dans chacune des perspectives, et non pas une neutralité "sans valeur» dans la démarche théorique et des valeurs "réservées» dans la démarche pratique. Dans la mesure où se présente, pour un individu ou pour un groupe de théoriciens et de praticiens, une option à prendre "en faveur» de l'explication théorique et objective ou "en faveur" d'un engagement réformateur ou correctif de situations «jugées» moralement ou politiquement, il y a bien là une visée de valeurs. Le choix qui donne priorité à la constatation puis à l'analyse empirique et finalement à la théorisation est un choix originellement compromis avec ce qu'il prétend exclure, à savoir l'option judicative. Ceci vaut tout autant à propos du choix du paradigme quà propos du choix de l'opération rationalisante. Il faut reconnaître la même compromission avec ce qui est prétendument exclu chez les réformateurs, qui ne peuvent exclure des représentations et des perceptions de la situation qui est à corriger et jugée comme telle à partir d'une connaissance et d'un «diagnostic» opérant à l'intérieur d'un paradigme.

Dans chacune de ces insistances, théoriques et pratiques, il s'agira encore de relier des éléments distincts, et ce lien constitue le contenu même des deux démarches. Dans l'insistance théorique, sociologique en particulier, la dualité qui caractérise la question de l'aliénation est celle des states of mind et des structures objectives. Ces deux dimensions permettent de définir respectivement une aliénation subjective correspondant à des expériences personnelles d'ordre psychologique (M. SEEMAN, 1959) et une aliénation objective correspondant à la situation sociale (F. CHA- 
ZEL, 1976). Dans la première dimension, on tente de définir des sentiments, d'en préciser la teneur et surtout de les mesurer (par l'intermédiaire de leurs expressions dans les discours et les comportements). On retiendra, par exemple, les sentiments de dépersonnalisation, d'incertitude, d'incompréhension, d'absurdité, d'isolement, de frustration, de rejet, etc. La sociologie empirique américaine a particulièrement développé la recherche en ce domaine (D.G. DEAN, L. SROLE, K. KENISTON, R. BLAUNER). Dans la seconde dimension, on tente de localiser les conditions d'émergence de ces sentiments dans les structures sociales et d'établir des corrélations (statistiques) entre des comportements caractérisés et leurs environnements objectifs. La sociologie marxiste s'est largement orientée vers de telles analyses $^{2}$.

\section{IL Y A UNE VISÉE PRATIQUE}

Dans l'insistance pratique, la dualité est celle des fondements et des stratégies. Il s'agit de réaliser dans et par l'organisation de structures socio-politiques et économiques des valeurs justifiant des interventions, des organisations et des comportements aussi bien chez les intervenants eux-mêmes que chez ceux pour qui et au nom de qui l'intervention s'effectue. «Liberté, égalité, fraternité» et l'abolition de la monarchie; "prolétaires de tous les pays, unissez-vous» et l'abolition du capitalisme pour l'avènement du communisme, voilà des valeurs et des stratégies d'abolition, de remplacement, d'instauration de nouvelles structures qui devraient «réelliser» ces valeurs. Dans la plupart des projets politiques, ce sont des valeurs morales qui sont citées, en dernière instance ou implicitement, comme justifications de l'abolition de situations objectives ou de l'instauration de nouvelles structures plus «efficaces» non seulement à l'obtention de ce qui est voulu, mais surtout à l'obtention de ce qui «doit» être voulu. Certains prétendent, et non sans raison, que les projets de Marx aussi bien que ceux de Durkheim, soucieux de fonder des interventions désaliénantes, étaient essentiellement des projets moraux (J. HORTON, 1964, A. SCHAFF, 1970, R. GARAUDY, 1964, M. RUBEL, 1970). Pour sa part, Georg Lukacs

2. On trouvera un bilan intéressant de cette situation dans F. GEYER \& D. SCHWEITZER, eds, Theories of Alienation, Leiden, M. Nijhoff, 1976. 
fonde explicitement le socialisme sur la morale (G. LUKACS, article posthume, 1979). L'insuffisance des convictions personnelles des promoteurs de réformes socio-politiques, et leur impuissance à convaincre de l'obligation inconditionnelle de réaliser certaines valeurs aussi bien que leur impuissance à convaincre de la nécessité de certains modes particuliers, privilégiés, d'intervention en faveur d'une telle réalisation de valeurs, ont conduit plusieurs à tenter de justifier "scientifiquement" des projets éthico-politiques.

À partir de la conviction que l'homme, individuel et collectif, a le devoir d'être et de faire certaines choses pour faire respecter ce qui est aussi son droit, on a voulu, depuis Platon, soustraire ce devoir de l'arbitraire en le fondant sur une connaissance des faits proprement caractéristiques du dynamisme, factuellement constaté, des hommes. Devant l'impossibilité, d'ailleurs rapidement concédée, d'orienter l'initiative libre tout en la laissant précisément «libre», la rhétorique politique et morale s'est confinée à la constatation de propensions "naturelles» en supposant, assez dogmatiquement, qu'il ne se poserait pas de question, et qu'il ne «devait» pas s'en poser «normalement» concernant l'obligation de faire ce que les hommes en général sont "spontanément" portés à faire et qui est socialement rentable. Établissant des besoins et des conditions inéluctables nécessaires à la satisfaction de ces besoins, on a cru pouvoir dépasser le dogmatisme, associé à l'idéologie, des perspectives déontologiques, en localisant un telos inscrit dans le mouvement de l'histoire, de la psychologie individuelle, de la culture, de la biologie.

Un modèle éidétique chez Platon, l'homme travailleur chez Marx, le vivant chez Nietzsche, une personnalité intégrée chez Freud, Maslow, Fromm, un porteur de gène chez Monod et plus récemment chez Laborit et $\mathrm{E}$.W. Wilson, c'est à une dimension factuelle, constatée ou représentée comme indépendante d'une initiative «libre» du sujet humain, que l'on fait appel pour déterminer ce que l'homme devrait être et ce qu'il devrait faire pour réaliser des aspirations «naturelles», i.e. fondées sur une réalité décrétée comme «norme» de ces devoirs. Si la désaliénation et les interventions qu'elle exige sont «fondées" sur une réalité normative de cet ordre, il faut bien admettre alors qu'un autre fondement s'imposera dès qu'un individu (ou une collecti- 
vité) plus exigeant que les autres réclamera des raisons de se soumettre de façon signifiante à des processus psychologiques, biologiques, historiques qui ne sont, somme toute, que des faits eux-mêmes à justifier par un fondement qui serait ultime, un fondement sans fondement, i.e. qui n'en aurait pas besoin pour l'être.

\section{IL FAUT INTERVENIR}

Ces fondements constituent les normes du diagnostic qui déterminera non seulement les valeurs (par implication), mais surtout les «thérapies» ${ }^{3}$. Ceci nous permet d'analyser maintenant la seconde dimension de l'insistance pratique: la stratégie d'intervention. La situation étant perçue comme urgente, pénible et généralisée, les interventions se portent vers le plus "provoquant» de l'aliénation et vers les éléments considérés (avec une naïveté déconcertante dans bien des cas) comme les "causes» ou les occasions immédiates, accessibles à l'intervention structurale. Les appels plus ou moins clairement formulés ne manquent pas pour inciter à intervenir, et généralement ils fonctionnent comme des catalyseurs de propensions psychologiques altruistes ou de réactions spontanées à la souffrance ou devant elle. Faute en effet d'avoir pu démontrer que les hommes avaient l'obligation, assumée par eux-mêmes, d'intervenir pour réaliser une valeur au sujet de laquelle ils n'auraient pas, et avec raison, à se demander pourquoi ils la viseraient, les incitateurs n'ont d'alternative pour amener ceux-ci à intervenir qu'à le faire en «jouant» (de façon plus ou moins sincère) sur leurs propensions «naturelles», leurs besoins, leurs tendances, leurs réflexes. Ces interventions, conditionnées par l'état d'esprit des intervenants aussi bien que par l'habileté rhétorique des incitateurs, porteront sur des structures et sur des «responsables» auxquels on ne se sera pas adressé, les ayant définitivement classés comme «irrécupérables», ou chez lesquels les valeurs promues par l'incitation n'auront pas eu l'effet d'engagement attendu.

C'est ainsi que des structures, des comportements, des modes d'organisation du travail, des modes de participation

3. L'aliénation est souvent associée à la maladie. E. FROMM oppose le terme «sain" à celui d'«aliéné» (1969); D. CRAYBROOKE utilise les termes "diagnostic et remèdes» (1958) On considère généralement l'aliénation mentale comme une maládie mentale plutôt que comme son effet social. 
politique, des organisations institutionnelles et politiques, des systèmes de distribution du pouvoir seront visés, soit pour être éliminés, soit pour être instaurés ou transformés afin d'en finir avec l'aliénation réduite alors à des expériences d'insatisfaction ou à des situations dans lesquelles des droits, ou des aspirations considérés comme tels, ne sont pas ou sont insuffisamment respectés. On tente alors d'organiser des "libérations» (qui sont en fait des soulagements de besoins factuellement constatés ou vécus) en fournissant aussi bien l'encadrement théorique ou idéologique que les modes «rationnels» politiquement efficaces de réalisation.

Il s'agit en quelque sorte de "planifier", si ce n'est, littéralement, de "contrôler» ce qui est prétendu être la réalisation objective, observable et même définitive des personnes et des collectivités pour garantir la reconnaissance concrète du statut de personne dans et par des modes universalisés de rapports intersubjectifs et de rapports collectifs aux produits humains. La définition que Marx donne du communisme à l'époque des Manuscrits (1844) est à ce titre exemplaire, sinon de l'ensemble des préoccupations de Marx lui-même, du moins des attitudes fréquentes de ceux qui en ont assez de théoriser sur les solutions et ont «décidé» d'intervenir à partir d'un «décret».

Ce communisme ( . . ) est la vraie solution du conflit de l'homme avec l'homme, la vraie solution de la lutte entre l'existence et l'essence, entre l'objectification et l'affirmation de soi, entre la liberté et la nécessité, entre l'individu et l'espèce. Il est l'énigme de l'histoire résolue et il sait qu'il est cette solution ${ }^{4}$.

Si Marx reconnaît que c'est l'histoire qui fera surgir cette solution, il reconnaît aussi que l'on peut «abréger la période de gestation» et que le capital (et non le capitaliste individuel) ne s'inquiétera pas de la santé et de la durée de vie du travailleur (comme si «le capital» pouvait faire quelque chose comme «s'inquiéter»!) «s'il n'y est pas contraint par la société» 5 .

\section{ON PEUT RÉUSSIR}

Les deux insistances, théorique et pratique, comportent chacune une prétention plus ou moins clairement reconnue. Dans

4. K. MARX, Économie et philosopbie, in Oeuvres, «économie», Paris, La Pléiade, tome II, 1968, p. 79.

5. K. MARX, Le capital, livre I, in Oeuvres, Paris, La Pléiade, 1965, t. I, p. 550 et 806 , 
l'insistance théorique, la prétention est qu'il y a une «rationalité» telle dans l'aliénation qu'il serait possible de prévoir son surgissement dans l'expérience et même de déduire la réalité de l'aliénation à partir des situations objectives (propriété privée, dépendance politique ou économique, ostracisme culturel, modes de pensée ou de comportement), et cela même dans les cas où aucune «expérience» consciente de «déperdition» de soi n'est vécue ou déclarée. Si cette prétention conserve l'avantage de permettre d'engager la démarche scientifique, la prétention de l'insistance pratique, elle, me semble beaucoup moins positive.

Les intervenants dans l'entreprise de désaliénation concrète prétendent qu'il est possible de donner ou de redonner, à un individu qui l'aurait soi-disant "perdu", son statut d'humain complet objectif de façon à contraindre "logiquement" (ou à imposer à leur perception "réaliste») tous les autres humains à "constater" la réalité de ce statut, et ce, avec la conviction que la reconnaissance concrète de ce statut, par l'intermédiaire du respect des devoirs que ce statut "entraîne", s'effectuera "automatiquement ${ }^{6}$. Cette prétention comporte également la conviction qu'on peut «obtenir» le statut d'humain autonome et libre par une position objective dans une structure, et qu'on peut l'obtenir également par la modification autoritaire et contraignante du comportement des autres envers les individus aliénés. Bref, la prétention qu'on pourrait se passer de la libre initiative, incontrôlable et, disons-le sans jouer sur les mots, «inaliénable» des autres pour faire reconnaitre effectivement et réellement le statut de personne à tous les individus humains.

Statuts politique, économique, juridique, social, culturel exprimeront alors les formes «objectives» et suffisantes de reconnaissance du statut de personne, et ce, à travers des droits, des privilèges, des attitudes observables, des performances et des pouvoirs qui seront reconnus ou accordés à des individus comme «preuves» de leur réalité «personnelle».

On connaît bien les échecs de la prétention théorique. La critique d'un savoir neutre et objectif de style empirico-déductif

6. Des lacunes dans la justification des interventions n'impliquent pas qu'il n'y ait pas de justification adéquate. Il s'agit très précisément ici de certaines justifications et des stratégies qu'elles commandent pour la réalisation de valeurs insuffisamment «faondamentales». 
sur l'homme s'est développée rapidement depuis des courants aussi différents que l'existentialisme et la "théorie critique» de l'École de Francfort. La compromission idéologique des «sciences» de l'homme a été particulièrement mise en évidence dans les objections suscitées par le structuralisme, le behaviorisme et la sociologie empirique américaine (en occurrence la sociobiologie récente de E.O. WILSON) et dans la radicalisation des questions soulevées par ce que l'on a appelé la «crise du marxisme», particulièrement évidente en France depuis mai 1968. Par ailleurs, la prétention théorique a subi des critiques internes au plan des méthodes et de l'ambiguïté des concepts, spécialement en ce qui concerne l'aliénation ( L. FEUER, 1962, L. SCAFF, 1964, T. OVEREND, 1975, H. LEFEBVRE, 1975, J. ISRAEL, 1976).

On connaît également les échecs de la prétention politique de désaliénation, surtout en milieu socialiste. Certains parlent même de «l'utopie de la désaliénation» (S. STOJANOVIC, 1969). La pensée yougoslave a insisté depuis G. LUKACS sur le caractère fétichiste d'une société parfaite où l'épanouissement des individus serait organisé, planifié, technicisé, garanti (O. GRUENDWALD, 1977). La critique de la désaliénation autoritaire menée par l'élite révolutionnaire s'appuie sur la nécessité d'une dialectique qui laisserait à l'individu la possibilité de se réaliser selon son originalité dans un avenir et une situation «ouverts» plutôt que selon un modèle unique et figé qui brime l'initiative personnelle (c'était déjà le voeue de Marx). C'est ainsi que A. HELLER donne à la philosophie radicale une fonction de «défétichisation» (1979). En France, André GLUCKSMANN a fait la critique de la révolution au nom de la révolte (in $\mathrm{F}$. CHATELET, 1978) et Joseph GABEL a défini même l'aliénation comme une «dédialectisation» (1970).

\section{QUI EST ALIÉNÉ?}

Ce qui, à mon avis, semble particulièrement négligé, c'est la question des conditions de possibilité de l'aliénation réelle, c'està-dire de l'absence réelle (vécue comme telle et/ou objectivement reconnaissable) d'un statut concret qui serait démontré comme un droit légitime à partir de la «monstration» du devoir et de l'obligation morale correspondant à ce droit. La recherche de ces conditions de possibilité nous amène à mettre en évidence les 
facteurs qui limitent la validité des entreprises aussi bien des chercheurs que des réformateurs, et ce, en montrant que ces entreprises sont inaugurées à partir de et dans une situation qui est elle-même une situation d'aliénation. Cette auto-implication dans le problème compromet l'objectivité de la description, le diagnostic et l'interprétation des effets et des facteurs déterminants de certaines situations dites aliénantes.

Faute de pouvoir ou de vouloir tenir compte des expériences vécues immédiates des intéressés (R.D. LAING, 1973), l'observateur et l'intervenant sont privés d'une connaissance «falsifiable» et adéquate de ce qui se passe réellement au plan des effets affectifs et des expériences internes de la subjectivité des acteurs ( $C$. MOREUX, 1978:48), bref des expériences qui permettent justement à l'acteur de s'attribuer à lui-même le statut de conscience et de personne. On soupçonnera alors que le diagnostic extérieur d'aliénation pourrait bien être l'expression d'une interprétation «aliénée» au sens que les observateurs théoriciens donnent euxmêmes fréquemment à ce terme: absence d'un lien indispensable à certaines réalités pour l'actualisation des «besoins» spécifiquement humains. L'aliénation des observateurs et des intervenants est ici proprement un «délire», une fiction inaperçue, mais déterminant des conceptualisations et des interventions auxquelles manque la propriété essentielle qui en ferait des entreprises non aliénées, à savoir la propriété d'être des décisions assumées de soi-même pour des objectifs fondamentaux connus.

Si l'on peut savoir de quoi sont privées certaines personnes ou certains groupes, si l'on peut savoir ce qui leur est factuellement nuisible au plan de la satisfaction physiologique, psychologique, culturelle ou politique, il semble beaucoup plus difficile de savoir si quelqu'un est, effectivement et objectivement, privé de sa "réalité» comme personne, comme "humain", comme fondement de droits, comme condition d'un agir libre. Les termes qui servent le plus généralement à qualifier la position des êtres aliénés évoquent, sinon une essence humaine, au moins un statut dans la hiérarchie des réalités, hiérarchie implicitement déterminée par le "traitement» subi par des humains et qui les «réduit» de façon prétenduement «efficace» à un statut inférieur: réifé, chosifié, déshumanisé, dépersonnalisé, réduit à l'état d'instrument, de facticité absurde, de donné sans initiative propre et 
sans spontanéité, dépossédé, considéré comme «abstraction», etc. Pour savoir qu'un individu humain «est» effectivement aliéné de cette façon, plutôt que, par exemple, être en train de subir des tentatives de réduction aliénante ou de "penser» qu'il est ainsi réduit réellement dès qu'une tentative surgit de le réduire, il faudrait pouvoir déterminer des critères objectifs permettant de reconnaître la réalité du statut de personne, au-delà des privilèges socio-politiques ou économiques accordés comme signes «suffisants» d'un statut, octroyé à tous par un décret que l'on ne justifie par aucune vérification dans l'expérience. Le statut social de personne semble suffire en général pour confirmer le statut ontologique de personne parce que l'on semble réduire les aspirations à la considération objective de soi comme personne plutôt qu'à la réalisation de soi comme personne ${ }^{7}$. Si une telle considération devait être nécessaire, il faudrait qu'elle repose sur une initiative signifiante du considérant et une réalité déjà effective du considéré comme «considérable».

Plusieurs sont tellement assurés de leurs critères d'aliénation qu'ils n'hésitent pas à parler d'une aliénation «inconsciente». Il s'agirait, dans l'aliénation, non pas tant d'une conscience du manque que d'un manque de conscience (G. FRIEDMAN, 1964: 203). Lorsque des individus ne se plaignent pas de leur sort dans des situations décrétées «objectivement» aliénantes (parfois à partir d'une compulsion émotionnelle qui s'exprime en terme de «sens moral»), d'autres parlent d'une baisse du niveau d'aspiration, de processus d'accoutumance, de satisfaction aliénée, etc. (R. BLAUNER, 1964; cf. H. STUCK, 1969).

On distingue assez mal en général la compassion qui s'impose à la conscience envers les souffrances d'un individu ou envers les privations qui semblent dévaloriser (ou favoriser une dévalorisation) des individus ou des groupes, et le diagnostic d'aliénation (J. HAJDA, 1961:758). La confusion est encore plus fréquente lorsqu'il s'agit d'un individu qui subit des comportements méprisants, des rejets, des exclusions, des frustrations ou est confiné à

7. De nombreux individus semblent revendiquer des positions et des comportements exprimant le respect de leur statut d'être inaliénable sans tenir, apparemment et à court terme, à s'assurer des intentions (intérêts) qui détermineraient ces comportements envers eux. On semble fréquemment vouloir se satisfaire d'un "signe" de reconnaissance plutôt que d'une reconnaissance justifiée par la réalité objective du stazut à reconnaître. Serait-il possible que nous ne croyions pas nous-mêmes à la réalité que nous demandons aux autres de reconnaître? 
des travaux ou à des positions sociales et qu'on ne voit pas comment cela serait compatible avec l'appropriation et la solidarité dont on remarque plus les effets "spontanément» désirables qu'on n'en voit les fondements justificateurs. C'est ainsi que MARX, après avoir déclaré dans l'introduction au Capital que son point de vue "peut moins que tout autre rendre l'individu responsable de rapports dont il reste socialement la créature, quoi qu'il puisse faire pour s'en dégager», conclut le livre I sur un ton particulièrement révélateur d'un changement de perspective, à moins que l'histoire des catégories économiques n'ait des «mobiles»!

L'expropriation des productions immédiats s'exécute avec un vandalisme impitoyable qu'aiguillonnent les mobiles les plus infâmes, les passions les plus sordides et les plus haïssables dans leur petitesse ${ }^{8}$.

Des individus ou des groupes (le "peuple»), considérés ou traités «objectivement» comme des machines ou des esclaves au service d'un patron, d'un propriétaire, d'un système, d'un État, d'un ordre hiérarchique qui les maintient dans l'ignorance et l'impuissance, sont considérés "aliénés». Cette aliénation a même été récemment conçue comme le point de départ qui permettrait de combattre la méconnaissance essentielle qui marque la perception du "vulgaire» sur son illusoire liberté et sa fictive subjectivité, et de vaincre la domination du système social, et ce, par la découverte, dans l'aliénation, de l'impossible et impuissante «opinion personnelle». Cette découverte rendrait «réaliste» et amènerait le peuple à "choisir sa parole en choisissant ses portes paroles» (P. BOURDIEU, 1979:489; cf. P. RAYNAUD, 1980: 90-92).

\section{LE DIAGNOSTIC RENVERSÉ}

S'il est, d'une certaine façon, justifiable de considérer que le témoignage de l'individu sur lui-même ne constitue pas une explication mais fait partie en quelque sorte du phénomène à expliquer, il reste que, d'une part, la position de l'individu dans une situation ou une structure et, d'autre part, l'absence constatable de valeurs, de privilèges, de pouvoirs, ne sont pas des prémis-

8. K. MARX, Le capital, op. cit, p. 550 et 1238. 
ses suffisantes pour conclure à l'irréalité du statut de personne. En tirant malgré tout une telle conclusion, les analystes et les révolutionnaires prennent pour acquis que le statut d'humain et la valeur humaine essentielle ne peuvent subsister sans des objectivations précises indispensables, sans une position précise dans une structure économique ou politique, sans des comportements, des privilèges, des pouvoirs, des connaissances, des sensations et même des aspirations jugés, d'après je ne sais quelle révélation, comme indispensables. On argumente comme si certains besoins constituaient le critère certain de démarcation entre les personnes (qui peuvent et veulent les satisfaire) et ceux qui ne le sont pas (qui ne peuvent et ne veulent pas les satisfaire). Ces inéluctables voies de "réellisation" de la personnalité constituent des "fétiches" dont on attend magiquement et dogmatiquement qu'ils produiront nécessairement la reconnaissance effective du statut de personne et même fourniront ce statut. Le refus souvent intempestif de justifier des «évidences morales", pourtant contestées et contestables, concernant les «devoir être» et les «devoir faire» manifeste, à mon avis, des attitudes magiques, animistes et dogmatiques qui sont elles-mêmes des symptômes d'une dépendance de la pensée d'une certaine façon «aliénée», i.e. conditionnée par des facteurs «autres» qui échappent à la libre initiative et déterminent ainsi un mode d'existence et de comportement sans fondement personnel.

En constatant que des valeurs s'imposent à l'esprit des chercheurs et des révolutionnaires, qu'elles ne sont pas le résultat d'une décision dont le caractère autonome soit clair et indubitable, qu'elles constituent une propension factuelle, réduisant les agents à être des exécutants qui n'ont pas une connaissance suffisante des valeurs fondamentales pour garantir des initiatives assumées de façon signifiante, i.e. qui soient à la fois libres et fondées, on doit les considérer comme «aliénantes».

En effet on considère fréquemment que l'exécution d'un travail dont on n'a pas été le promoteur, dont on ne connaît pas le sens, que l'on fait par compulsion et dont le produit n'est pas le nôtre, est une aliénation. Dès lors la réalisation d'une valeur, que ce soit une vérité ou un mode de société, pourrait être une aliénation si elle n'est pas le résultat d'un agir par soi-même, mais un projet factuellement mais inconsciemment imposé on ne sait 
d'où, et dont nous serions les exécutants irresponsables mais satisfaits.

Plusieurs travailleurs manuels considèrent que les théoriciens sont aliénés non seulement par la distance qui sépare ces derniers d'eux-mêmes (les théoriciens sont des marginaux, des exclus, des anormaux), mais également en tant que coupés de la réalité quotidienne, déshumanisés, désincarnés, privés des dimensions jugées essentielles d'une vie vraiment humaine. Inversement, des théoriciens considèrent que des travaux dont celui qui les exécute ne voit pas le sens, ou des impuissances au plan de l'autodétermination, ou des dévalorisations culturelles, économiques, politiques et psychologiques, sont des indices d'aliénation, sinon des signes d'aliénation. D'une certaine façon donc l'aliénation est diagnostiquée et donne lieu, éventuellement, à des engagements réformateurs à partir d'une position qui est ellemême aliénée.

Ce retour critique des intervenants et des théoriciens sur eux-mêmes permet d'envisager la possibilité d'une stratégie plus modérée, plus efficace et moins sujette à ce que Raymond BOUDON appelle «les effets pervers», i.e. les effets opposés à ceux qui sont attendus. On a mené depuis un certain temps des critiques semblables en science avec des résultats qui s'annoncent fort satisfaisants (K.R. POPPER, I. PRIGOGINE, P. FEYERABEND, etc.). Par ailleurs, dans la foulée du projet d'une «science» de l'idéologie, on a vu se développer une critique de la rationalité qui a permis de mettre en évidence les «ruses de la raison» se critiquant elle-même de son propre point de vue en récupérant ainsi constamment son «autre». En philosophie, en politique, en histoire, en psychologie, en sociologie et en anthropologie, c'est le projet même d'une critique de la «sciencificité» des sciences dites «humaines" qui a mis à jour une aliénation de la raison (DERRIDA, FOUCAULT, KOLAKOWSKI, HABERMAS, MARCUSE, ADORNO, LAING). Dans la mesure où l'aliénation est un concept qui a pu trouver sa place dans ces disciplines, il est bien évident que la "science» même de l'aliénation s'est trouvée impliquée dans son objet et est devenue plus "réflexive» qu'elle ne voulait l'admettre. Claude LEFORT (1955:68) et John HORTON (1964:295) ont bien mis en évidence la position problématique des théorisations et des projets 
de correction de l'aliénation. Erich FROMM (1969) parle même de critères sociaux d'aliénation qui seraient eux-mêmes des indices d'une société aliénée. C. WRIGHT MILLS (1966-184-188), dans un chapitre de son analyse des Cols Blancs, intitulé «la société anonyme des cerveaux», prétend que les recherches théoriques sur l'aliénation sont des indices d'aliénation, les chercheurs étant privés des moyens d'agir efficacement et fuyant dans ce qu'il appelle «la comédie de l'objectivité» ou le «fétichisme de l'objectivité et de l'aliénation». De même P. BERGER et PULLBERG expriment en termes très clairs cette situation:

Theoretical reifications, expressive of pre-reflexive and pretheoretical reifying consciousness can themselves become reified ${ }^{9}$.

\section{LES CONDITIONS NÉCESSAIRES DE L'ALIÉNATION RÉELLE}

De la situation analysée plus haut on peut, à mon avis, tirer le double constat suivant: une analyse non aliénée de l'aliénation n'est pas possible à moins de pouvoir établir les conditions de possibilité et les critères de réalisation d'un agir par soi-même et une connaissance de la signification «finale» de cet agir chez celui-là même qui entreprend l'analyse. D'autre part, une approche théorique non aliénante n'est pas possible non plus, étant donné la visée impersonnelle de la théorie aussi bien que ses méthodes impersonnalisantes. «On ne peut connaître quelque chose des hommes, a écrit L. ALTHUSSER, qu'à la condition absolue de réduire en cendre le mythe philosophique (théorique) de l'homme ${ }^{10}$.» $\mathrm{Si}$ une approche ne pouvait être ni aliénée ni aliénante, ce ne pourrait être qu'une approche inaugurée par un être non aliéné et pour un objectif signifiant et connu dans toute la transparence qu'exige un acte posé par un sujet "de lui-même» ${ }^{11}$. Un robot programmé pour mépriser quelqu'un ou en nier la réalité (comme un "capital» obéissant à des lois assimilables à celles de la nature, tel que présenté par MARX!) ne pourrait réussir qu’à réaliser un programme de manifestations ou de signes (interprétés comme tels par une "victime»), mais il ne pourrait lui-même dévaloriser et aliéner quelqu'un lui-même, bien qu'il puisse conditionner une

9. P. BERGER \& S. PULLBERG, "Reification and the Sociological Critique of Consciousness", in New Left Review, 1966, Feb., p. 66.

10. L. ALTHUSSER, Pour Marx, Paris, Maspero, 1977, p. 236.

11. G. LANE, «Pourquoi il n'y a pas d'obligation», communication ACP, 1980. 
croyance à cette négation de soi (perçue comme intentionnelle) si la victime ignore qu'il s'agit d'un robot n'agissant pas par luimême, mais répondant à un déterminisme absurde ou venu d'ailleurs.

Pour que l'analyse théorique de l'aliénation des individus aussi bien que l'entreprise de thérapie de certaines formes d'aliénation puissent éviter d'être aliénantes, il faudrait qu'elles soient, l'une et l'autre, inaugurées en faveur d'une réalité injustifiable et ultimement justificatrice, fondement dernier irréductible à ce qui est chose, objet, matière, ou encore irréductible à ce qui est étape, moment de processus, occasion, instrument, etc. La seule réalité qui me semble répondre à cette définition est la "personne», non pas telle qu'elle est prétendue simplement par chacun des hommes, mais peut-être telle qu'elle est visée et espérée par ceux qui, on ne sait encore pourquoi exactement, la pressentent comme réalité ultime. Pour viser ainsi des personnes, il faudrait que celles-ci soient reconnues comme telles, sinon dans les faits empiriques, du moins en tant que sujets d'un droit à la "personnalité». Une fois cette reconnaissance réalisée, on devra admettre que l'individu qui «est» une personne ne peut plus perdre ce statut qui le définit lui-même à moins de n'être plus ce «lui-même» mais un «autre». On pourra encore «tenter» de lui enlever ce statut, on ne pourra réussir que si notre entreprise n'est pas aliénée (comme celle d'un être sans volonté personnelle propre) et que s'il y consent de lui-même puisqu'il détient cette capacité d'agir de lui-même. (On ne voit pas évidemment pourquoi une personne renoncerait d'elle-même à être une personne, mais du point de vue formel cela est possible.)

Cette estime «justifiée» de soi comme personne détentrice légitime du droit à la reconnaissance et à l'inaliénabilité n'est pas nécessairement acquise une fois pour toutes, mais peut être réalisée dans une relation humaine particulière de façon suffisamment intense pour que des mépris, des humiliations, des travaux absurdes deviennent pratiquement inefficaces à affecter le statut d'un individu conscient d'être inaliénable, en droit et en fait, même lorsque ce statut n'est pas, ou pas assez, clairement reconnu. Ceci évidemment n'empêchera pas de souffrir, de la méconnaissance d'un statut légitime et essentiel, mais ne le fera pas disparầ- 
tre comme semblent le laisser entendre ceux qui utilisent les termes de «réification" ou de «chosification" 12 .

Dans la situation où nous sommes chacun individuellement, il ne semble pas possible d'établir objectivement, intersubjectivement et universellement notre existence comme personne et notre valeur sans «passer par» des structures et des performances qui justifieraient notre prétention à valoir comme personne plutôt que comme chose ou «élément» d'un système, d'une nation, d'une structure. Le refus des «ismes» chez certains philosophes, la haine des «maittres penseurs» chez les nouveaux philosophes, les réactions «individualistes» devant la montée du nationalisme et jusqu'à la non-directivité en pédagogie (P. FEYERABEND, 1979) et en psychiatrie (T. SZASZ, 1974; P. FEDIDA, 1977) témoignent de très diverses manières de tentatives de «jouer» à l'intérieur des structures pour protéger la singularité personnelle et sa «spontanéité» créatrice. On connaît bien, par ailleurs, l'inéluctabilité culturelle de la récupération par la classification (M. FOUCAULT, 1972; J. BAUDRILLARD, 1972).

Ce «passage» et cette récupération dans les structures sont souvent confondus avec la volonté d'obtenir une valorisation par ou au moyen des structures et performances plutôt qu'à l'occasion ou dans les structures ou performances. L'idée même d'une situation objectivement aliénante que défendent certains sociologues n'est pas sans évoquer l'idée corrélative de situations objectivement désaliénantes. Par ailleurs, les revendications de ceux qui souffrent de frustrations concernant leur intégration sociale ou leurs rapports à leurs propres produits (État aussi bien que marchandises) sont fréquemment sélectionnées par les grands «libérateurs" au profit de l'instauration de structures que nous avons associés plus haut au fétichisme, structures qui sont prétendues désaliénantes à des niveaux d'expérience qui sont parfois fort différents de ceux dans lesquels se vivent les frustrations ${ }^{13}$.

12. Cela ne signifie pas que l'aliénation ne pourrait être réduite que par des expériences «individuelles» vécues dans l'intimité d'une relation "privée». Des sous-groupes, dont le nombre de participants peut être très grand, peuvent fournir des expériences de reconnaissance de soi permettant de "compenser" des méconnaissances subies dans d'autres sousgroupes, par exemple: les homosexuels, les hippies, les femmes, les noirs, les émigrés.

13. Certaines idéologies syndicales sont fort loin de leurs idéaux lorsqu'elles proposent des stratégies économiques pour sauver la «dignité» des travailleurs. 
La fuite de la réification passe ainsi par une objectification qui risque à chaque entreprise de nous happer dans la choséité (inconscience, fétichisme, servilité, etc.), de manière à ce que le soupçon que nous sommes reconnus pour notre avoir, notre pouvoir, nos performances, nos fonctions plutôt que pour notre originalité et notre unicité personnelle est toujours présent. Il serait tentant, dans cette perspective, de penser que le renoncement à vouloir autre chose que ce que nous avons déjà, le renoncement à vouloir être autre que ce que nous sommes déjà (ou pensons être), le renoncement à vouloir valoir plus que ce que nous valons déjà (au moins pour certaines personnes), et même le renoncement à vouloir valoir quelque chose plutôt que de ne rien valoir objectivement, il serait tentant donc de penser que ces renoncements, à la Schopenhauer ou à l'orientale, seraient des désaliénations, des libérations de toutes les normes, des identifications «sereines» de soi tel qu'on est. Mais il semble que cette «solution» soit à la fois incohérente et inacceptable en vertu de l'expérience persistante de ce qui semble être un «devoir» d'accomplissement aussi bien qu'un «destin» d'inachèvement.

Les conditions de l'aliénation réelle sont donc d'ordre ontologique et social. Il faut qu'il existe des personnes détentrices d'un droit légitime à la reconnaissance en vertu d'une valeur propre et objective. Ces réalités personnelles doivent être susceptibles de perdre soit leur statut social, soit leur réalité «essentielle». Un processus ou une situation ne peuvent être efficacement aliénants que s'ils sont inaugurés par une réalité personnelle et privent réellement une ou d'autres personnes de leur statut ou de leur réalité. L'expérience d'aliénation suppose conscience et vouloir sans lesquels le diagnostic d'aliénation ne peut être justifié. Enfin, il faut admettre que la perte du statut social ou la méconnaissance de la valeur des personnes n'impliquent pas nécessairement la perte de la réalité de personne, réalité que l'on a peine à concevoir comme susceptible d'être aussi bien "perdue" que «donnée» ou reçue.

\section{UNE ALIENATION RÉSURGENTE}

Un certain niveau d'aspiration, et même certaines sortes d'aspirations sont considérés comme indispensables à une reconnaissance justifiée du statut de personne, ou, à tout le moins, sont 
jugés très exactement révélateurs de la "qualité» de ce statut. La différence entre les désirs aliénés et ceux qui ne le sont pas viendrait des objets désirés plutôt que du mode de désir. Ce que l'on ne remarque pas assez, à mon avis, c'est que l'augmentation du niveau d'aspiration aussi bien que le changement d'objet des aspirations n'indiquent pas une désaliénation, sinon au sens d'une victoire sur le rejet dévalorisant de soi par un groupe particulier d'une collectivité, mais plutôt une nouvelle aliénation. En effet, dans cette modification des aspirations se trouve éclipsée la question de la nécessité pour un être non aliéné d'agir de lui-même et d'être un soi-même qui ne résulte pas d'une soumission conditionnée à un modèle culturellement sanctionné de personne, même si cela est «gratifiant» ${ }^{14}$.

Par ailleurs, la «découverte» d'un idéal plus grand, plus exigeant au plan des performances, plus orienté vers l'autodétermination et vers la réalisation de valeurs qui transcendent telle ou telle matérialité de l'existence, cette découverte ne peut s'accompagner d'une plus grande satisfaction (associée à la désaliénation) qu'à la condition inéluctable d'être «modérée». Plus l'ambition grandit, plus l'idéal devient irréalisable, plus le souci d'autonomie devient intense et plus les doutes peuvent augmenter sur l'authenticité vécue par rapport à une autonomie totale, transparente et parfaite. Le «réalisme» auquel on fait appel fréquemment pour justifier cette modération n'est pas loin de la résignation et présuppose un inventaire définitif des moyens. Par ailleurs, les «fondements» biologiques de la morale qui devrait se limiter aux obligations imposées par la «nature» à l'espèce humaine (E.O. WILSON, 1979; H. LABORIT, 1970) évoquent une soumission aux normes naturelles que l'on «devrait» assumer alors que ces normes sont paradoxalement présentées comme «toujours déjà» à l'oeuvre dans toutes les initiatives humaines. Le problème se pose pour les dichotomies défendues par les moralistes entre le fait et le droit ou entre ce qui est et ce qui doit être (B.C. BIRCHALL, 1978). Un tel projet de se soumettre aux «bonnes» normes ne peut certes pas se présenter comme simplement désaliénant, mais peut-être comme plus efficacement satisfaisant à l'intérieur d'une aliénation perçue comme inéluctable sans trop d'explications ou de résistances.

14. Voir mon article "Usage logique, psychologique et idéologique de la notion de gratuité ", in Dialogue, XV, 3, septembre 1976, 394J-414. 
Dans la perspective pratique et réformatrice de style plus «politique» et où l'on vise à intervenir pour modifier la situation objective, culturelle, économique, psychologique et idéologique, on peut faire le même reproche d'une modification d'aliénation. La transformation des structures à cause de leurs propriétés dites aliénantes n'aboutit en effet le plus généralement qu'à la transformation d'une situation de dépendance pénible en une autre situation de dépendance qu'on espère ou décrète "bénéfique», mais qui est encore une dépendance du statut de personne envers des statuts politiques et sociaux accordés "de l'extérieur» par des structures, des capacités, des performances, des positions gratifiantes dans une hiérarchie de pouvoir et de «signes» de respect.

Les urgences d'une amélioration des modes d'existence sont bien réelles et les frustrations ou les souffrances liées aux structures économiques et politiques sont intenses et importantes. Nous ne prétendons nullement ici qu'il faudrait négliger les interventions qui visent le bien-être individuel et collectif à un plan bien concret. Mais si l'individu «doit» satisfaire des besoins avec comme seule raison que ces besoins sont là, factuels, réels, ressentis et pressants, il faut bien admettre qu'il ne s'agit plus de morale mais de «zoologie», comme le pensait Nietzsche, et qu'il ne s'agit pas non plus de valeur mais de compulsion absurde. Dans ces conditions, les opérations de gratifications ne sont pas vraiment désaliénantes, comme on le prétend, puisqu'elles ne fournissent pas aux "machines désirantes» un statut de personne, fondement de droit et capable d'agir par elles-mêmes comme des subjectivités libres. S'il est vrai que l'obligation (et non pas la contrainte ou la compulsion) d'assumer les valeurs morales et sociales comme la justice, l'égalité, la tolérance, la paix, la dignité et le respect des droits des «personnes» est une obligation qui concerne des personnes, il faudrait que les structures socio-économiques et politiques qui réaliseraient cette obligation dans les faits ne soient pas l'équivalent de la création d'un «zoo» humain permettant de fournir sécurité et nourriture aux animaux fragiles que seraient les hommes dans «l'état de nature» duquel ils ne pourraient s'échapper.

La désaliénation organisée et idéologiquement déterminée (aussi bien que «scientifiquement» justifiée par les exigences 
naturelles de l'homme) favorise donc une nouvelle aliénation par tout ce qu'elle implique de généralisation dogmatique de valeurs d'appréciation et par ses reconnaissances finalement conditionnelles du statut de personne et de la réalité personnelle. Une certaine homogénéisation (sous le couvert d'une certaine forme de «rationalisation» relevant d'un pragmatisme de courte vue) des critères de reconnaissance du statut de personne autonome, achevée, accomplie, en «pleine» possession d'elle-même, force les individus à répondre à un modèle de comportements et de pensées (d'aspirations, disions-nous plus haut) ou même à un modèle de sensations et d'expériences, et ce, pour être considérés et reconnus «effectivement» et objectivement comme personne authentique. Cette considération et cette reconnaissance se justifient dans les discours moraux et révolutionnaires par ce que les personnes sont déjà «fondamentalement" et «essentiellement» et à la fois par ce que, paradoxalement, elles ont aussi à être, à devenir, à faire. La contradiction est flagrante, et la confusion totale.

Les femmes constituent actuellement un exemple d'une telle modification d'aliénation lorsqu'elles se voient contraintes, pour certaines d'entre elles et de très diverses façons, d'adopter des valeurs nouvelles, socialement privilégiées, pour être appréciées et reconnues comme femmes désaliénées et "rendues" à ellesmêmes. Du même élan elles se voient interdire, psychologiquement ou «moralement», d'aimer et de se sentir même "gratifiées", comme si cela était impossible, par des fonctions, des pouvoirs, des positions et des aspirations devenus objectivement et nécessairement aliénants et qui ne l'étaient peut-être pas dans la situation existentielle concrète et individuelle (ou qui étaient peut-être le lieu d'une aliénation, mais non pas sa cause ou sa condition suffisante et nécessaire).

Il semble donc, à voir ce qui se passe, que la reconnaissance de soi comme être inconditionnellement digne de cette reconnaissance ne puisse se réaliser concrètement comme telle. C'est bien plutôt conditionnellement à des soumissions ou à des conformités à des modèles de performances ou de comportements ou d'expériences que la reconnaissance de la subjectivité personnelle pourra se manifester dans les faits. C'est ainsi, par exemple, que des travailleurs seront "acceptés» par des libérateurs à condition d'avoir «compris», d'être "conscients», de ressentir une obliga- 
tion "morale" envers leurs collègues, de vouloir des améliorations socio-politiques "positives", de ne pas être "complices" du pouvoir établi et de l'oppresseur, etc. On comprend ainsi comment peut avoir un sens l'expression contradictoire de «humain inhumain». Cette conformité ou cette absence de conformité à des modèles plus ou moins contraignants ou présentés comme tels déterminent l'orientation d'une désaliénation vers de nouvelles formes plus raffinées de «dépossession» ou, au mieux, vers des satisfactions qui jouent à l'intérieur d'une aliénation définitivement «concédée». Tout se passe comme si les personnes avaient à justifier, par des comportements et des performances précises, leur statut devant d'autres personnes qui se prétendent plus ou moins confusément détentrices des normes nécessaires, et qu'elles devaient d'autre part conquérir ce statut plutôt que d'en être simplement les bénéficiaires légitimes et évidents, ce que plusieurs énoncent d'ailleurs très dogmatiquement par une «foi» dans «l'homme».

En voulant que le statut de personne soit reconnu à tous (parce que des personnes inaliénables sont pourtant aliénées!), en voulant faire prendre conscience à ceux qui ne revendiquent pas le droit qu'ils ont, qu'ils l'ont «effectivement» et «naturellement» (c'est-à-dire f finalement: "évidemment»), soit encore en voulant modifier des situations qui constituent une dénégation objective de ce droit, on semble prétendre que tous auraient ce droit et que tous auraient l'obligation (plutôt que simplement la propension) qui correspond à ce droit ${ }^{15}$.

On suppose donc que celui qui m'aliène (directement ou par des détours idéologiques ou économiques) n'est pas un robot dont je pourrais changer le programme, mais qu'il est un être capable d'agir de lui-même et d'assumer de lui-même une obligation proprement morale envers moi-même. La manière de faire assumer ce devoir est un problème fondamental qui surgit au sein du procès des "urgences", mais il est clair qu'en forçant des comportements (par la modification de structures contraignantes), on a

15. Je rappelle encore une fois ici, parce que le sujet de ma réflexion est souvent l'occasion de réaction émotionnelle intempestive en faveur des "évidences morales", que la critique des justifications des entreprises théoriques et pratiques concernant l'aliénation n'implique pas de soi une réfutation de la valeur de ces entreprises elles-mêmes, mais vise des arguments insuffisants disponibles actuellement. 
éliminé le surgissement libre et signifiant, non aliéné, de ces comportements qu'on voudrait ne pas être aliénants, mais sans s'assurer qu'ils ne sont pas, aussi, aliénés, et finalement inefficaces.

Des facteurs de satisfaction de besoins semblent confondus avec des facteurs qu'on dit "d'humanisation» et de «désaliénation", comme si être humain c'était être heureux, satisfait, aussi bien au plan des aspirations économiques que politiques ou psychologiques. D'autre part, des satisfactions particulières sont dites aliénées lorsqu'elles ne sont pas celles d'un être humanisé et conscient. Il reste vrai cependant que l'on ne peut pas tout simplement reléguer les privations au second plan et considérer de manière très platonicienne ou ascétique que le statut de personne n'est en rien concerné par les relations avec la collectivité, la position sociale et les expériences émotionnelles. L'indifférence à la souffrance des autres n'est pas moins aliénée et aliénante que la pitié compulsive si rien n'intervient pour faire de ces attitudes des initiatives par soi-même.

L'effet de privations physiques, psychologiques, sociales, culturelles ou économiques n'est pas la négation du statut de personne, et leur apaisement ne constitue pas la confirmation de ce statut (W. KAUFFMANN, in R. SCHACHT, 1970: xxvii). Le sommeil ne fait pas de moi à tout point de vue un «objet», ni le mépris que je peux subir et dont je peux souffrir sans en devenir la victime vaincue. Dans la mesure où ce qui est affecté en moi n'est pas moi proprement et principalement, et dans la mesure où ce qui affecte mes sensations n'est pas d'origine libre et personnelle, n'est pas effectué par un être non aliéné agissant par lui-même, de lui-même, mais est plutôt le jouet de déterminismes qui le dépasssent, alors la situation ne peut être dite «objectivement» aliénante. Ce qui nous ferait retenir tout de même ce qualificatif, c'est l'expression collectivement ou individuellement significative d'une tentative, elle-même aliénée, de mépris et de dévalorisation.

J'irais même plus loin en disant que la volonté d'être une personne et d'être reconnue comme telle ne peut avoir de sens que si elle n'est pas factuelle, mais répond à une décision inaugurée par un être qui serait déjà une personne, d'une certaine façon 
«suffisamment» réelle pour être capable de vouloir par lui-même devenir un soi achevé comme identité, comme toujours le «même», bref comme un soi-même plutôt que «autre». Il faudrait donc que celui qui prétend me désaliéner me fournisse la capacité d'agir par moi-même, et il ne peut faire cela sans être déjà capable lui-même d'agir de lui-même. Comment cela peut-il commencer? Il semble que c'est là la question qui permettrait d'échapper au dogmatisme de la désaliénation qui résulte de l'ignorance dans laquelle nous nous trouvons des véritables fondements des interventions «morales» et politiques.

Je terminerai en disant que ce que Wallace MATSON a appelé «l'axiome de Frankenstein», qui dit que «toute reproduction physique exacte d'un être sentant (Sentience) sera lui-même un être sentant» (1976:81), conduit, dans la question de la désaliénation par la seule réorganisation objective des structures socio-économiques, politiques et mentales, à un monde de Frankenstein, un monde d'aliénés privés de leur identité propre et de leur initiative personnelle autonome, mais, d'une certaine façon, de la seule façon peut-être, heureux du bonheur des pierres.

On prétendra que le statut de personne est un «dû», mais il faudrait pouvoir établir, d'une part, la légitimité de la revendication de ce statut (sa «rentabilité» est déjà problématique) et, d'autre part, les conditions de possibilité de la satisfaction de cette revendication (le fondement du «devoir moral» des autres). Ce qui me semble important pour l'instant, c'est de remarquer que les désaliénations sont, dans la plupart des cas, des transformations d'aliénations pénibles en aliénations inaperçues et en quelque sorte mieux supportables, et quelquefois presque «satisfaisantes".

Département de philosophie

Collège Laflèche 


\section{BIBLIOGRAPHIE}

ADORNO, Theodor. Dialectique négative. Paris, Payot, 1978.

BAUDRILLARD, Jean. Pour une critique de l'économie politique du signe. Paris, Gallimard, 1972, coll. "Tel».

BERGER, P.L. et LUCKMANN, Th. The Social Construction of Reality. N.Y., Anchor Books, 1967.

BIRCHALL, B.C. «Moral Life as the Obstacle to the Development of Ethical Theory", Inquiry, 21, 4, Winter 1978.

BLAUNER, Robert. Alienation and Freedom. Chicago, Chicago Univ. Press, 1964.

BOURDIEU, Pierre. La distinction. Paris, Minuit, 1979.

BRAYBROOKE, David. «Diagnosis and Remedy in Marx's Doctrine of Alienation», Social Research, 1958, 325-345.

CRAWFORD, David R. "Interpretations of Self-Alienation», International Pbilosophical Quarterly, 16, 1976, 323-340.

DJILAS, Milovan. Une société imparfaite. Paris, Calmann-Lévy, 1969.

FEDIDA, Pierre. Le concept et la violence. Paris, Un. Gén. d'éd., 1977, coll $10 / 18$.

FEUER, Lewis. «What is Alienation: The Career of a Concept», New Politics, 1962, 1, 116-134.

FEYERABEND, Paul. Contre la méthode. Paris, Seuil, 1979.

FISHER, Claude S. "Alienation: Trying to Bridge the Chasm», British Journal of Sociology, 27, 1976, 1, 35-50.

FOUCAULT, Michel. Histoire de la folie à l'âge classique. Paris, Gallimard, 1972, coll. «Tel».

FRIEDMAN, Georges. Le travail en miettes. Paris, Gallimard, 1964.

FROMM, Erich. Société saine et société aliénée. Paris, Éd. Courrier du livre, 1969.

GABEL, Joseph. Sociologie de l'aliénation. Paris, P.U.F., 1970.

GARAUDY, Roger. Karl Marx. Paris, Seghers, 1964.

GLUCKSMANN, André. «Idéologie et révolte», in CHATELET Fr., éd., Histoire des idéologies, Paris, Hachette, 1978.

GRUENDWALD, Oskar. "The Concept of Alienation in Avant-Garde Yugoslav Marxism», International Pbilosopbical Quarterly, 17, 1977, 195-218.

HAJDA, Jan. "Alienation and Integration of Students Intellectuals», American Sociological Review, 26, 1961, 758-777.

HELLER, Agnes. Pour une philosopbie radicale. Paris, Sycomore, 1979.

HORTON, John. "The Dehumanization of Anomie and Alienation», British Journal of Sociology, 15, 1964, 283-300.

ISRAEL, Joachim. L'aliénation: de Marx à la sociologie contemporaine. Paris, Anthropos, 1972.

LABORIT, Henri. L'agressivité détournée. Paris, Union Gén. d'éd., 1970, coll $10 / 18$.

LAING, Ronald D. La politique de l'expérience. Paris, Stock, 1973. 
LANE, Gilles. Être et langage. Paris, Aubier, 1970.

Idem. L'Urgence du présent (essai sur la culture et la contre-culture). Montréal, P.U.Q., 1973.

Idem. "Il est impossible d'intervenir dans le cours des événements», Dialogue, $18,1979,1,1-13$.

LEFEBVRE, Henri. «Sur l'aliénation», Social Praxis, 3, 1975, 63-76.

LEFORT, Claude. "L'aliénation comme concept sociologique» (1955), in Les formes de l'bistoire, Paris, Gallimard, 1978.

LUKACS, Georg. "Le fondement moral du communisme», La Pensée, 206, 1979.

MATSON, Wallace. Sentience. California University Press, 1976.

MOREUX, Colette. La conviction idéologique. Montréal, P.U.Q., 1978.

MORIN, M. et BERTRAND, C. Le territoire imaginaire de la culture. Montréal, HMH, 1979.

OVEREND, Tronn. "Alienation: a Conceptual Analysis», Philosopbical and Phenomenological Research, 35, 1975, 3, 301-322.

RAYNAUD, Pierre. "Le sociologue contre le droit», Esprit, 1980, 3, mars.

ROSNER, Menachem. «Scientific concept or ideological weapon» (An argument about the concept of alienation), Revue Internationale de Sociologie, (Italie), 1971, no 2, part. 2, p. 958-977.

RUBEL, Maximilien. Pages de Karl Marx pour une étbique socialiste. Paris, Payot, 1970, coll P.B.P.

SCAFF, Laurence. "Conceptualizing Alienation: Reductionism and the Problem of Meaning", Philosophy of the Social Sciences, 8, 1978, 3, 241-260.

SCHACHT, Richard. Alienation Intro. by KAUFMANN, W., N.Y., Anchor Book, 1970.

SCHAFF, Adam. Marxism and the Human Individual. N.Y. McGraw Hill, 1970.

SEEMAN, Melvin. «On the Meaning of Alienation», American Soc. Review, 24, 1959, 6, 783-791.

Idem. "Alienation and Engagement", in CAMPBELL, A. \& CONVERSE, P.E., eds, The Human Meaning of Social Change, N.Y., Russell Sage, 1972, p. 467-527.

Idem. «Empirical Alienation Studies: an Overview», in GEYER, R.F. \& SCHWEITZER, D.R., Theories of Alienation, Leiden, Martinus Nijhoff, 1976.

STOJANOVIC, S. Between Ideals and Reality. N.Y., Oxford Univ. Press, 1973.

STUCK, Heiner. «Automation et aliénation», Épistémologie sociologique, 8, 1969, 89-119.

SZASZ, Thomas. Le mythe de la maladie mentale. Paris, Payot, 1974.

WILSON, Edward Osborne. L'bumaine nature. Paris, Stock, 1979.

WRIGHT MILLS, C. Les cols blancs. Paris, Seuil, 1966. 